\title{
COVID-19 and myocarditis/myocardial injury
}

\author{
(DKaan Okyay \\ Başkent University, Faculty of Medicine, Department of Cardiology, Ankara, Turkey
}

Cite this article as: Okyay K. COVID-19 and myocarditis/myocardial injury. J Health Sci Med 2021; 4(3): 388-390.

\begin{abstract}
Myocarditis is defined as inflammation of the myocardium, and it presents with different clinical pictures, and its diagnosis has some difficulties. The prognosis of myocarditis is aetiology-dependent, and treatment is fundemantally supportive except for "endometrial biopsy proven" cases which requires spesific therapies including immunomodulators and intravenous immunoglobulins. SARS-CoV-2 affects cardiovascular systems directly or via indirectly mechanisms. COVID-19 infection increases the risks of myocardial injury, myocarditis, myocardial infarction, heart failure, venous thromboembolism and arrhythmias. The underlying mechanism of troponin elevation during COVID-19 infections is not exactly explained. In this review, the relationship between COVID-19 and myocardial involvement was discussed. Still, multidisciplinary trials are needed to explain this important situation.
\end{abstract}

Keywords: COVID-19 infection, myocardial injury, myocarditis

Myocarditis is defined as inflammation of the myocardium, and it presents with different clinical pictures, and its diagnosis has some difficulties. The clinical scenario differs from asymptomatic status to subtle symptoms such as weakness, fatigue, and overt cardiac symptoms including chest pain and palpitation, and even to (sudden) death due to cardiogenic shock and malignant ventricular arrhythmias. The prognosis of myocarditis is aetiology-dependent. It may completely resolve without any spesific therapy particulary in patients having mild symptoms with normal ventricular functions. However, one third of the cases may develop dilated cardiomyopathy which could be irreversibl after therapy, on the other hand, it is possible that the ventricles do not ameliorate resulting in overt heart failure. The treatment is fundemantally supportive but in rare "endometrial biopsy proven" cases, spesific therapies including immunomodulators and intravenous immunoglobulins may be applied $(1,2)$. SARS-CoV-2 affects cardiovascular systems directly or via indirectly mechanisms. The virus attaches to the ACE2 receptor in many human cells including type 2 alveolar cells of the lung, myocardial cells, vascular endothelial cells, kidney proximal tubule cells, gastrointestinal cells and vesical cells, and has direct cytotoxic effects. Involvement of myocytes and vascular endothelial cells results in myocardial injury. Furthermore, cytokine storm secondary to inflammation, increased sympathetic activity, epicardial coronary thrombus due to tendency to caogulation, microvascular thrombus, pulmonary embolism and stres induced cardiomyopathy are related with myocardial injury. Eventually, COVID-19 infection increases the risks of myocardial injury, myocarditis, even myocardial infarction, heart failure, venous thromboembolism and arrhythmias. As seen in many different conditions, elevation in blood cardiac troponin levels only reflect myocardial injury. However, the underlying mechanism of troponin elevation during COVID-19 infections is not exactly explained. The presence of intensive inflammation; so called cytokine storm; can emerge troponin release stand alone. Nevertheless, rare cases of fulminant myocarditis are reported.

The other argued mechanisms of troponin elevation are type-1 myocardial infarction related with coronary plaque rupture due triggered prothrombotic system activition, and type- 2 myocardial infarction related with imbalance between oxygen demand and supply. In the contemporary literature, there are no data regarding echocardiography and particulary cardiac MR findings in patients with elevated cardiac troponin levels. So, the relationship between cardiac troponin elevation and myocardial structural and functional abnormalities is equivocal. Accordingly, troponin 
elevation seen during COVID-19 infections does not mean acute coronary syndrome (ACS). For diagnosis of ACS, other clinical findings should be observed (ECG and echocardiographic changes, and symptoms). Nonetheless, independent of the underlying reason, increases in cardiac troponins predict poor prognosis. After initial echocardiography, the most useful imaging tool for myocarditis is cardiac MR.

The cost, relatively longer process time and need for stability and cooperation of the patient are the limitations. Alternatively, cardiac CT is introduced to show myocardial involvement. In COVID-19 patients, differantial diagnosis of ACS-myocarditis-secondary myocardial inferction is possible using cardiac CT imaging. Although it aids in certain diagnosis, endomyocardial biopsy is very rare performed. Histopathology reveals myocyte degeneration along with inflammatory infiltration and non-ischemic necrotic zones. In suspicion of COVID-19 related myocarditis, routine use of steroids is not recommended. In a spesific patients group with cytokine storm immonuspression is proven to have beneficial effects. Also, there is not any spesific antiviral agent for prevention as well as treatment of myocarditis. Hence, cases vith myocarditis should be followed up with supportive therapies and should be closely monitored for development of heart failure and arrhythmias. In progressive disease despite heart failure therapies and in patients with fulminant myocarditis which developt end organ failure, there are case reports on the usage of myocardial assist devices and extracorporeal membrane oxygenation (3-8). There is no doubt that further multidisciplinary trials are needed regarding relationship between COVID-19 and myocarditis.

At the end of this short review, two recent important studies on the cardiac biopsy and cardiac MR findings of the patients with COVID-19 infection will be mentioned. In a autopsy trial of Pellegrini et al (9) from Italy, 40 hearts from subjects dying of COVID-19 infection (mainly due to respiratory failure) were investigated. One of the third subjects had evidence of myocyte necrosis, predominantly of the left ventricle. The major cause of myocyte necrosis was microthrombi in $2 / 3$ of the cases which were distinct in composition (i.e. greater fibrin and c5b-9 complement) as compared to intramyocardial thromboemboli from COVID-19 negative subjects and to coronary thrombi aspirated from COVID-19 positive and negative STEMI patients. The authors have concluded that the clinicians should be aware of the microthrombi which may not be detectable clinically as a cause of cardiac injury in COVID-19 patients and pointed out the importance of tailored anti-thrombotic strategies to counteract the effects of microthrombi on the heart. In a cardiac MR study of Kotecha et al (10), 148 patients from 6 centers in England with severe COVID-19 infection (all requiring hospital admission, 1/3 requiring ventilatory support) and troponin elevation discharged alive underwent cardiac MR at median 68 days. Left ventricular function was normal in $89 \%$ of the patients. Late gadolinium enhancement and/or ischaemia was found in 54\% (myocarditis-like scar in 26\%, infarction and/or ischaemia in $22 \%$ and dual pathology in 6\%). Myocarditis-like injury was limited to three or less myocardial segments in $88 \%$ of cases with no LV dysfunction; of these, $30 \%$ had active myocarditis. Interestingly, among patients with ischaemic injury pattern, $66 \%$ had no history of coronary artery disease. The authors have remarked that furher and robust trials are needed to investigate the relationship of these MR findings with clinical course of the disease.

In conclusion, COVID-19 infection increases the risks of myocardial injury and myocarditis. Even in the absence of COVID-19 infection, the diagnosis of the myocarditis inherently has some difficulties. In the clinical picture of COVID-19 infection, there are many factors related with cardiac troponin elevation. In this point, a careful and comprehensive evaluation of the possible mechanism is of great importance.

\section{ETHICAL DECLARATIONS}

Referee Evaluation Process: Externally peer-reviewed.

Conflict of Interest Statement: The authors have no conflicts of interest to declare.

Financial Disclosure: The authors declared that this study has received no financial support.

Author Contributions: All of the authors declare that they have all participated in the design, execution, and analysis of the paper, and that they have approved the final version.

\section{REFERENCES}

1. Turkish Cardiology Association Consensus Report: COVID-19 Pandemic and Cardiovascular Diseases. Turk Kardiyol Dern Ars 2020; 48: 1-87.

2. Siripanthong B, Nazarian S, Muser D, et al. Recognizing COVID-19-related myocarditis: the possible pathophysiology and proposed guideline for diagnosis and management. Heart Rhythm 2020; 17: 1463-71

3. Guo T, Fan Y, Chen M, et al. Cardiovascular implications of fatal outcomes of patients with coronavirus disease 2019 (COVID-19). JAMA Cardiol 2020; 5: 811-8.

4. Hendren NS, Drazner MH, Bozkurt B and Cooper LT, Jr. Description and proposed management of the acute COVID-19 cardiovascular syndrome. Circulation 2020; 141: 1903-14.

5. Pirzada A, Mokhtar AT, Moeller AD. COVID-19 and myocarditis: what do we know so far? CJC Open 2020; 2: 278-85. 
6. Kawakami R, Sakamoto A, Kawai K, et al. Pathological evidence for SARS-CoV-2 as a cause of myocarditis. J Am Coll Cardiol 2021; 77: 314-25.

7. Okyay K, Sadıç BÖ, Şahinarslan A, et al. Turkish Society of Cardiology consensus paper on the rational use of cardiac troponins in daily practice. Anatol J Cardiol 2019; 21: 331-44.

8. Skulstad H, Cosyns B, Popescu BA, et al. EACVI recommendations on precautions, indications, prioritization, and protection for patients and healthcare personnel. Eur Heart J Cardiovasc Imaging 2020; 21: 592-8.

9. Pellegrini D, Kawakami R, Guagliumi G, et al. Microthrombi as a major cause of cardiac injury in COVID-19: a pathologic study. Circulation 2021; 143: 1031-42.

10. Kotecha T, Knight DS, Razvi Y, et al. Patterns of myocardial injury in recovered troponin-positive COVID-19 patients assessed by cardiovascular magnetic resonance. Eur Heart J 2021:ehab075. 